Document downloaded from:

http://hdl.handle.net/10251/106299

This paper must be cited as:

Cordero Barbero, A.; García-Maimo, J.; Torregrosa Sánchez, JR.; Vassileva, MP. (2017). Multidimensional stability analysis of a family of bi-parametric iterative methods. Journal of Mathematical Chemistry. 55(7):1461-1480. doi:10.1007/s10910-016-0724-6

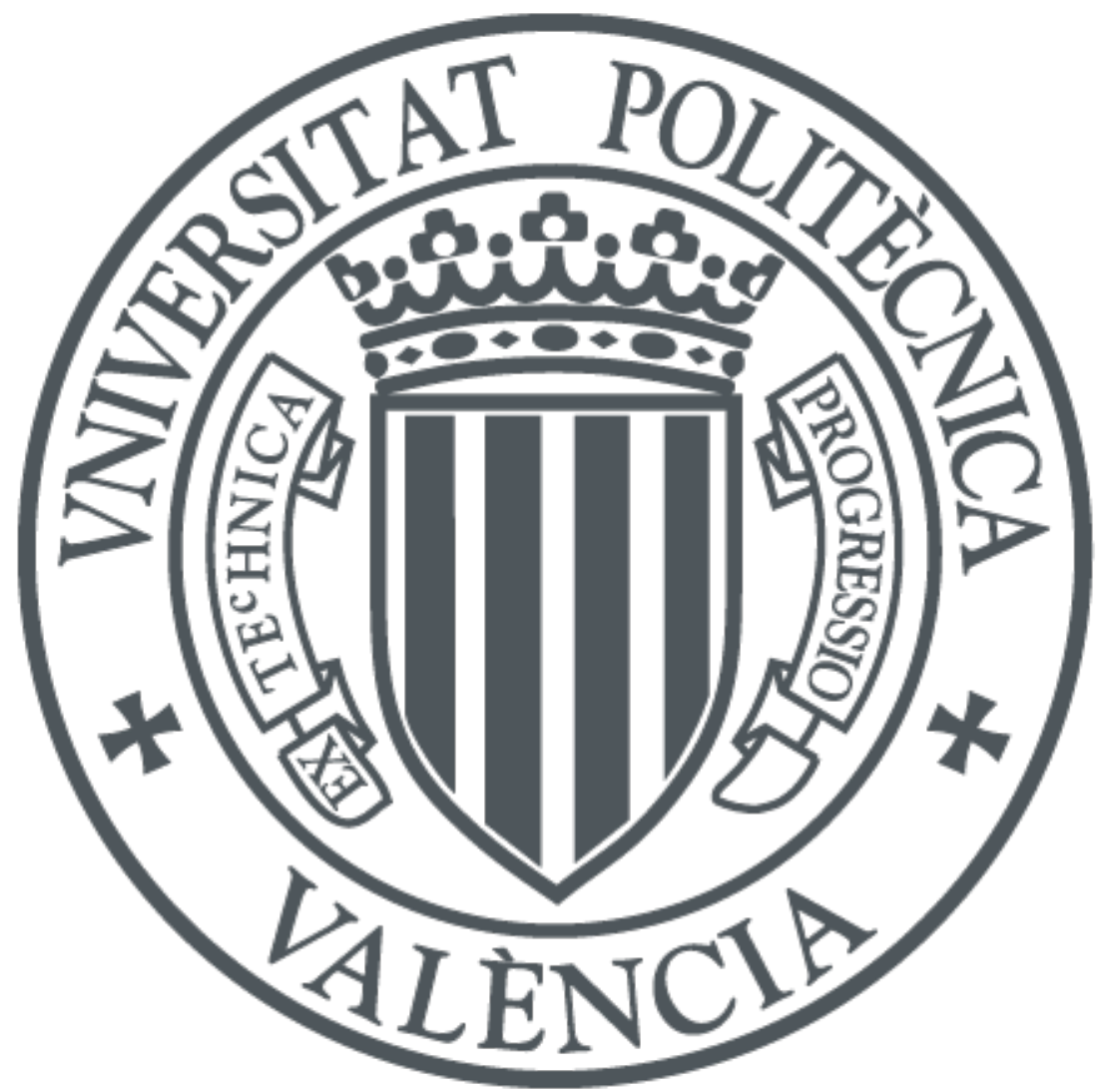

The final publication is available at

http://doi.org/10.1007/s10910-016-0724-6

Copyright Springer-Verlag

Additional Information 


\title{
Multidimensional stability analysis of a family of biparametric iterative methods - CMMSE2016
}

\author{
Alicia Cordero • Javier G. Maimó · Juan R. Torregrosa • \\ María P. Vassileva
}

Received: date / Accepted: date

\begin{abstract}
In this paper, we present a multidimensional real dynamical study of the Ostrowsky-Chun family of iterative methods to solve systems of nonlinear equations. This family was defined initially for solving scalar equations but, in general, scalar methods can be transferred to make them suitable to solve nonlinear systems. The complex dynamical behavior of the rational operator associated to a scalar method applied to low-degree polynomials has shown to be an efficient tool for analyzing the stability and reliability of the methods. However, a good scalar dynamical behavior does not guarantee a good one in multidimensional case. We found different real intervals where both parameters can be defined assuring a completely stable performance and also other regions where it is dangerous to select any of the parameters, as undesirable behavior as attracting elements that are not solution of the problem to be solved appear. This performance is checked on a problem of chemical wave propagation, Fisher's equation, where the difference in numerical results provided by those elements of the class with good stability properties and those showed to be unstable, is clear.
\end{abstract}

Keywords Nonlinear system of equations · Iterative method · Basin of attraction · Dynamical plane · Stability · Fisher's equation

\section{Introduction}

Let us consider the problem of finding a real zero of a function $F: D \subseteq \mathbb{R}^{n} \longrightarrow \mathbb{R}^{n}$ that is, a solution $\bar{x} \in D$ of the nonlinear system $F(x)=0$, of $n$ equations with $n$ variables, being $f_{i}, i=1,2, \ldots, n$ the coordinate functions of $F$. This solution can be obtained as a fixed point of some function $\bar{G}: \mathbb{R}^{n} \longrightarrow \mathbb{R}^{n}$ by means of the fixed-point iteration method

$$
x^{(k+1)}=\bar{G}\left(x^{(k)}\right), \quad k=0,1, \ldots
$$

where $x^{(0)}$ is the initial estimation.

This research was partially supported by Ministerio de Economía y Competitividad MTM2014-52016-C02-2-P and FONDOCYT 2014-1C1-088 República Dominicana.

\section{Alicia Cordero}

Instituto de Matemática Multidisciplinar, Universitat Politècnica de València,

Camino de Vera, s/n, 46022 Valencia, Spain

E-mail: acordero@mat.upv.es

Javier G. Maimó

Instituto Tecnológico de Santo Domingo (INTEC),

Santo Domingo, Dominican Republic

E-mail: javier.garcia@intec.edu.do

Juan R. Torregrosa

Instituto de Matemática Multidisciplinar, Universitat Politècnica de València, Camino de Vera, s/n, 46022 Valencia, Spain

E-mail: jrtorre@mat.upv.es

Maria P. Vassileva

Instituto Tecnológico de Santo Domingo (INTEC),

Santo Domingo, Dominican Republic

E-mail: maria.penkova@intec.edu.do 
Methods for solving nonlinear equations $f(x)=0, f: I \subseteq \mathbb{R} \longrightarrow \mathbb{R}$ can be transferred to systems $F(x)=0$, $F: D \subseteq \mathbb{R}^{n} \longrightarrow \mathbb{R}^{n}$. The extension of a scalar method to multidimensional case requires to rewrite the iterative expression in such a way that there are no evaluations of the nonlinear function $f$ in the denominator, as they will become vectors in the extension to systems. To solve this problem the divided difference operator can be used $[x, y ; F]$, see [1]. Once the method has been transferred to multivariate case, a dynamical study can be made to see if good methods for solving nonlinear equations are still stable when extended to systems.

In [1] the Ostrowski-Chun family of iterative biparametric methods is introduced, and this class is extended for solving nonlinear systems by using the divided difference operator. Its iterative expression is

$$
\begin{aligned}
y^{(k)} & =x^{(k)}-\left[F^{\prime}\left(x^{(k)}\right)\right]^{-1} F\left(x^{(k)}\right), \\
x^{(k+1)}= & y^{(k)}-G\left(x^{(k)}, y^{(k)}\right)\left[F^{\prime}\left(x^{(k)}\right)\right]^{-1} F\left(y^{(k)}\right), \\
G\left(x^{(k)}, y^{(k)}\right)= & \frac{1}{a_{1}}\left[\left(1+a_{1} b_{2}-2 a_{1}\right) I-a_{1}\left(b_{2}-2\right)\left[F^{\prime}\left(x^{(k)}\right)\right]^{-1}\left[x^{(k)}, y^{(k)} ; F\right]\right]^{-1} \\
& +\frac{1}{a_{1}}\left(\left(a_{1}+a_{1} b_{2}-1\right) I-a_{1} b_{2}\left[F^{\prime}\left(x^{(k)}\right)\right]^{-1}\left[x^{(k)}, y^{(k)} ; F\right]\right),
\end{aligned}
$$

where $y^{(k)}$ is Newton's step, $\left[x^{(k)}, y^{(k)} ; F\right]$ is the divided difference operator of $F$ on $x^{(k)}$ and $y^{(k)}, I$ is the identity matrix and $F^{\prime}\left(x^{(k)}\right)$ is the Jacobian matrix of the system.

In this paper, we analyze the real multidimensional dynamical behavior of the family, in order to get information about the stability of the resulting schemes when suitable values of the parameters are selected. Depending on the intervals where they are defined, completely different performance can be found, and to detect these stable and unstable behaviors is a key fact in order to apply the members of this class for solving specific problems.

This paper is organized as follows: in Section 2 some concepts of real multidimensional dynamics are introduced. In Section 3 we study the dynamical behavior of the family of Ostrowski-Chun iterative schemes, finding the subintervals of the domain of the parameters where strange fixed points appear and their stability are analyzed in these cases with the aid of bifurcation diagrams. To get this aim, dynamical and convergence planes are successfully used in Section 4. To check the performance of some elements of the family, those are tested numerically on Fisher's equation in Section 5. Finally, in Section 6 some conclusions are stated.

\section{Basic concepts}

Some dynamical studies by using complex dynamics tools have been made for scalar iterative methods for solving nonlinear equations on low degree polynomials, see, for instance, [2-4]. These techniques have proved to be efficient to analyze the stability of a method or to select the most stable members of a family.

In this work, we propose a real multidimensional dynamical study of the Ostrowski-Chun family of iterative methods for solving systems of nonlinear equations. We analyze the dynamical behavior of a fixed-point iterative method for nonlinear systems when applied to a n-variable polynomial $p(x), p: \mathbb{R}^{n} \rightarrow \mathbb{R}^{n}, x \in \mathbb{R}^{n}$, by using the procedure stablished in [5]. We will start recalling some basic dynamical concepts; to deep in these concepts see, for example, the text [6].

Definition 1 Let $G: \mathbb{R}^{n} \rightarrow \mathbb{R}^{n}$ be a vectorial rational fixed-point function associated to the iterative method on polynomial $p(x)$. The orbit of a point $x^{(0)} \in \mathbb{R}^{n}$ is defined as the set of successive images of $x^{(0)}$ by $G(x)$, $\left\{x^{(0)}, G\left(x^{(0)}\right), \ldots, G^{m}\left(x^{(0)}\right), \ldots\right\}$.

We can classify the dynamical behavior of a point of $\mathbb{R}^{n}$ examining its asymptotic behavior. Hence, $x^{*} \in \mathbb{R}^{n}$ is a fixed point of $G$ if $G\left(x^{*}\right)=x^{*}$.

Definition 2 A periodic point $x$ of period $k \geq 1$ is a point such that $G^{k}(x)=x$ and $G^{k-p}(x) \neq x$, for $p<k$.

To check the stability of fixed or periodic fixed points for nonlinear operators we recall a known result in real discrete dynamics.

Theorem 1 ([6], page 558) Let $G$ from $\mathbb{R}^{n}$ to $\mathbb{R}^{n}$ be $\mathcal{C}^{2}$. Assume $x^{*}$ is a period- $k$ point. Let $\lambda_{1}, \lambda_{1}, \ldots, \lambda_{n}$ be the eigenvalues of $G^{\prime}\left(x^{*}\right)$.

a) If all the eigenvalues $\lambda_{j}$ have $\left|\lambda_{j}\right|<1$, then $x^{*}$ is attracting.

b) If one eigenvalue $\lambda_{j_{0}}$ has $\left|\lambda_{j_{0}}\right|>1$, then $x^{*}$ is unstable, that is, repelling or saddle. 
c) If all the eigenvalues $\lambda_{j}$ have $\left|\lambda_{j}\right|>1$, then $x^{*}$ is repelling.

Fixed points that are not a root of the polynomial $p(x)$ are called strange fixed points. If $x^{*}$ is an attracting fixed point of the rational function $G$, its basin of attraction $\mathcal{A}\left(x^{*}\right)$ is defined as the set of pre-images of any order such that

$$
\mathcal{A}\left(x^{*}\right)=\left\{x^{(0)} \in \mathbb{R}^{n}: G^{m}\left(x^{(0)}\right) \rightarrow x^{*}, m \rightarrow \infty\right\} .
$$

The set of points whose orbits tend to an attracting fixed point $x^{*}$ is called Fatou set, $\mathcal{F}(G)$, while the complementary set, the closure of the set consisting of its repelling fixed points that establishes the borders between the basins of attraction, is called Julia set, $\mathcal{J}(G)$

\section{Dynamical study of Ostrowski-Chun family of methods}

In this section, we apply the previous dynamical concepts to the multidimensional rational function associated 4th-order Ostrowski-Chun family of methods designed in [1], whose iterative expression appears in (2).

In particular, we will analyze the dynamical behavior of these family of methods acting on the polynomial system $p(x)=0$, where

$$
\left.\begin{array}{l}
p_{1}(x)=x_{1}^{2}-1 \\
p_{2}(x)=x_{2}^{2}-1
\end{array}\right\}
$$

By applying the iterative expression of Ostrowski-Chun class (2) on $p(x)$, we get its associate multidimensional rational function; later we study its real fixed points in order to analyze their stability.

The $j$ th-coordinate of the vectorial rational function associated to Ostrowski-Chun family on polynomial $p(x)$ is

$$
\lambda_{j}^{p}(x)=\frac{1}{8 x_{j}^{3}}\left(\frac{\left(b_{2}-2\right)\left(x_{j}^{2}-1\right)^{3}}{a_{1}\left(b_{2}-2\right)\left(x_{j}^{2}-1\right)+4 x_{j}^{2}}-\frac{b_{2}\left(x_{j}^{2}-1\right)^{3}+4 x_{j}^{2}-12\left(x_{j}^{2}+2\right) x_{j}^{4}}{4 x_{j}^{2}}\right), \quad j=1,2 .
$$

As fixed points are the solutions of $\bar{\lambda}_{j}^{p}(x)=x_{j}$, that is,

$$
\frac{1}{8 x_{j}^{3}}\left(\frac{\left(b_{2}-2\right)\left(x_{j}^{2}-1\right)^{3}}{a_{1}\left(b_{2}-2\right)\left(x_{j}^{2}-1\right)+4 x_{j}^{2}}-\frac{b_{2}\left(x_{j}^{2}-1\right)^{3}+4 x_{j}^{2}-12\left(x_{j}^{2}+2\right) x_{j}^{4}}{4 x_{j}^{2}}\right)=x_{j}, j=1,2,
$$

or, in a equivalent form,

$$
\begin{gathered}
\left(x_{j}-1\right)\left(x_{j}+1\right)\left(x_{j}^{6}\left(a_{1} b_{2}^{2}+18 a_{1} b_{2}-40 a_{1}+88\right)+x_{j}^{4}\left(-3 a_{1} b_{2}^{2}-18 a_{1} b_{2}+48 a_{1}-32\right)+\right. \\
\left.+x_{j}^{2}\left(3 a_{1} b_{2}^{2}-2 a_{1} b_{2}-8 a_{1}+8\right)-a_{1} b_{2}^{2}+2 a_{1} b_{2}\right)=0, j=1,2 .
\end{gathered}
$$

the following result can be formulated.

Proposition 1 The fixed points of the vectorial rational function associated to Ostrowski-Chun class on polynomial $p(x)$ are the roots of $p(x),(1,1),(1,-1),(-1,1)$ and $(-1,-1)$ and also the pairs $\left(r_{i}, r_{j}\right), i, j \in\{1,2, \ldots, 6\}$, whose entries are roots of the polynomial

$$
\begin{aligned}
r\left(x, a_{1}, b_{2}\right)= & x^{6}\left(a_{1} b_{2}^{2}+18 a_{1} b_{2}-40 a_{1}+88\right)+x^{4}\left(-3 a_{1} b_{2}^{2}-18 a_{1} b_{2}+48 a_{1}-32\right)+ \\
& +x^{2}\left(3 a_{1} b_{2}^{2}-2 a_{1} b_{2}-8 a_{1}+8\right)-a_{1} b_{2}^{2}+2 a_{1} b_{2} .
\end{aligned}
$$

These are strange fixed points that depend on $a_{1}$ and $b_{2}$.

Let us remark that $x_{j}=1$ and $x_{j}=-1$ always satisfy expression (4). As we have two possible values for $j$, that leads to the fixed points $(1,1),(1,-1),(-1,1)$ and $(-1,-1)$, the roots of $p(x)$.

To calculate the rest of fixed points, as they are the roots of $r\left(x, a_{1}, b_{2}\right)=0$ that has only even powers, a simple change of variables $t=x^{2}$ transform it in a 3rd-degree polynomial

$$
p_{2}(t)=(t-1) a_{1}\left(2\left(9 t^{2}-1\right) b_{2}+(t-1)^{2} b_{2}^{2}+8 t(1-5 t)\right)+8 t\left(11 t^{2}-4 t+1\right),
$$

whose roots $r_{21}, r_{22}$ and $r_{23}$, can be calculated by exact procedures. Then the components of the strange fixed points are $r_{i} \in\left\{ \pm \sqrt{r_{21}}, \pm \sqrt{r_{22}}, \pm \sqrt{r_{23}}\right\}, i \in\{1,2, \ldots, 6\}$. Moreover, we are interested only in the real strange fixed points, so depending on the values of the parameters we can have from none to 6 real different entries 
(besides of 1 and -1) of the vectorial strange fixed points. Therefore, strange fixed points are the combinations of the roots of $r$ with themselves and with 1 and -1 . So if only one root is real we have 12 strange fixed points, 32 if two roots are real and 60 in case all three roots are real.

The number of real strange fixed points depends on the values of $a_{1}$ and $b_{2}$. We get the real roots of $r$ depending on the value of $b_{2}$ first, and then analyzing each case depending on the value of $a_{1}$.

1. If $b_{2}<\frac{1}{63}(-820-176 \sqrt{22})$, then the number of strange fixed points depends on $a_{1}$ and also on the roots $r_{1 j}, j=1,2,3,4$ of $p_{1}(t)=t^{4}\left(b_{2}-2\right)^{4}\left(4 b_{2}-1\right)-2 t^{3}\left(b_{2}-2\right)^{3}\left(3 b_{2}+1\right)+t^{2}\left(b_{2}-2\right)^{2}\left(27 b_{2}^{2}-42 b_{2}+11\right)-$ $4 t\left(8 b_{2}^{2}-25 b_{2}+18\right)+28$ and of $r_{2 j}, j=1,2,3$ that are the roots of $p_{2}(t)$.

a) If $a_{1}<r_{11}$ or $a_{1}>r_{12}$, then there exist six real different entries of the strange fixed points $\pm \sqrt{r_{21}}, \pm \sqrt{r_{22}}, \pm \sqrt{r_{23}}$, giving a total of sixty points.

b) For $a_{1}=r_{11}$ or $a_{1}=r_{12}$, there are four possible real entries $\pm \sqrt{r_{21}}$ and $\pm \sqrt{r_{23}}$ or $\pm \sqrt{r_{22}}$, respectively.

c) If $r_{11}<a_{1}<\frac{-88}{-40+18 b_{2}+b_{2}^{2}}$ or $0 \leq a_{1}<r_{12}$ then there are only two real entries, $\pm \sqrt{r_{21}}$.

d) When $\frac{-88}{-40+18 b_{2}+b_{2}^{2}} \leq a_{1}<0$, there not exist strange fixed points.

2. For $b_{2}=\frac{1}{63}(-820-176 \sqrt{22})$, different cases must be analyzed:

a) If $a_{1}<\frac{-88}{-40+18 b_{2}+b_{2}^{2}}$ or $a_{1}>r_{12}$, then there exist six real possible entries for the strange fixed points, $\pm \sqrt{r_{21}}, \pm \sqrt{r_{22}}, \pm \sqrt{r_{23}}$.

b) If $a_{1}=r_{12}$, there are four real different entries $\pm \sqrt{r_{21}}, \pm \sqrt{r_{22}}$.

c) For $a_{1}=\frac{-88}{-40+18 b_{2}+b_{2}^{2}}$ or $0 \leq a_{1}<r_{12}$, there are only two real entries, $\pm \sqrt{r_{21}}$.

d) When $\frac{-88}{-40+18 b_{2}+b_{2}^{2}}<a_{1}<0$, there are not strange fixed points.

3. For $\frac{1}{63}(-820-176 \sqrt{22})<b_{2}<-20$, there are several possibilities:

a) If $a_{1}<\frac{-88}{-40+18 b_{2}+b_{2}^{2}}$ or $a_{1}>r_{12}$, there are six different entries for the strange fixed points: $\pm \sqrt{r_{21}}, \pm \sqrt{r_{22}}, \pm \sqrt{r_{23}}$.

b) When $a_{1}=\frac{-88}{-40+18 b_{2}+b_{2}^{2}} \leq a_{1}<r_{11}$ or $a_{1}=r_{12}$, there are four different real entries, $\pm \sqrt{r_{22}}$ and $\pm \sqrt{r_{21}}$ or $\pm \sqrt{r_{23}}$, respectively.

c) If $a_{1}=r_{11}$ or $0 \leq a_{1}<r_{12}$ there exist only two different entries, that is $\pm \sqrt{r_{22}}$ or $\pm \sqrt{r_{21}}$, respectively.

d) For $r_{11}<a_{1}<0$ there are not strange fixed points.

4. The number of real strange fixed points for $b_{2}=-20$ depends on the relative position of parameter $a_{1}$ and the roots of polynomials $p_{3}=4743684 t^{4}+314116 t^{3}-1409771 t^{2}+3718 t-7$ and $p_{2}(t)$, denoted by $r_{31}, r_{32}$ and $r_{2 j}, j=1,2,3$, respectively.

a) For $a_{1}>r_{32}$, there are six real entries $\pm \sqrt{r_{21}}, \pm \sqrt{r_{22}}, \pm \sqrt{r_{23}}$.

b) If $a_{1}<r_{31}$ of $a_{1}=r_{32}$, then $\pm \sqrt{r_{22}}, \pm \sqrt{r_{23}}$ or $\pm \sqrt{r_{21}}, \pm \sqrt{r_{22}}$, respectively, are the real entries of the strange fixed points.

c) When $a_{1}=r_{31}$ or $0 \leq a_{1}<r_{32}$, there exist two real entries $\pm \sqrt{r_{22}}$ or $\pm \sqrt{r_{21}}$, respectively.

d) For $r_{31}<a_{1}<0$ there not exist strange fixed points.

5. If $b_{2}$ is defined in the range $-20<b_{2}<-\frac{184}{29}$, then:

a) If $r_{12}<a_{1}<\frac{-88}{-40+18 b_{2}+b_{2}^{2}}$ then there are six real entries for the strange fixed points, $\pm \sqrt{r_{21}}, \pm \sqrt{r_{22}}, \pm \sqrt{r_{23}}$.

b) For $a_{1}<r_{11}$ or $a_{1}>\frac{-88}{-40+18 b_{2}+b_{2}^{2}}$, the real entries are $\pm \sqrt{r_{22}}, \pm \sqrt{r_{23}}$.

c) When $a_{1}=r_{12}$ or $a_{1}=\frac{-88}{-40+18 b_{2}+b_{2}^{2}}$, the real entries of the strange fixed points $\pm \sqrt{r_{21}}, \pm \sqrt{r_{22}}$.

d) If $a_{1}=r_{11}$ or $0 \leq a_{1}<r_{12}$ then there are only two real entries, $\pm \sqrt{r_{22}}$ or $\pm \sqrt{r_{21}}$, respectively.

e) For $r_{11}<a_{1}<0$, there not exist strange fixed points.

6. When $b_{2}=-\frac{184}{29}$, the number of real entries of the fixed points depends again on $a_{1}$ and also on the first root of polynomial $p_{4}(t)=602330740 t^{3}+563854192 t^{2}-11941127 t+170723$, denoted by $r_{41}$ and on $r_{2 j}$ $j=1,2,3$ that denote the roots of $p_{2}(t)$.

a) If $a_{1}<r_{41}$ or $a_{1}>\frac{841}{1089}$, then the real entries are $\pm \sqrt{r_{22}}, \pm \sqrt{r_{23}}$. 
b) For $a_{1}=r_{41}$ or $0 \leq a_{1} \leq \frac{841}{1089}$, there are only two real entries: $\pm \sqrt{r_{22}}$ or $\pm \sqrt{r_{21}}$, respectively. In particular, the case corresponds to the entry $\pm \sqrt{\frac{23}{59}}$.

c) When $r_{41}<a_{1}<0$, there are not strange fixed points.

7. If $-\frac{184}{29}<b_{2}<0$,

a) When $a_{1}<r_{11}$ or $a_{1}>\frac{-88}{-40+18 b_{2}+b_{2}^{2}}$, the real entries of the strange fixed points are $\pm \sqrt{r_{22}}, \pm \sqrt{r_{23}}$.

b) If $a_{1}=r_{11}$ or $a_{1}=\frac{-88}{-40+18 b_{2}+b_{2}^{2}}$ then the only real entries are $\pm \sqrt{r_{22}}$.

c) For $0 \leq a_{1}<r_{12}$ or $r_{12} \leq a_{1}<\frac{-88}{-40+18 b_{2}+b_{2}^{2}}$ there are also two real entries, that correspond to $\pm \sqrt{r_{21}}$ or $\pm \sqrt{r_{23}}$, respectively.

d) If $r_{11}<a_{1}<0$,there there are not strange fixed points.

8. For the case $b_{2}=0$, the number of real entries of the strange fixed points depend on $a_{1}$ and also on the roots of $p_{2}(t)$, denoted by $r_{2 j} j=1,2,3$.

a) If $a_{1}<-1-\sqrt{2}$ or $a_{1}>\frac{11}{5}$, then the number of real different entries is six: $\pm \sqrt{r_{21}}, \pm \sqrt{r_{22}}, \pm \sqrt{r_{23}}$

b) When $a_{1}=-1-\sqrt{2}$ or $1<a_{1}<\frac{11}{5}$, there are four real entries, $\pm \sqrt{r_{22}}$ and $\pm \sqrt{r_{21}}$ or $\pm \sqrt{r_{23}}$, respectively.

c) If $-1-\sqrt{2}<a_{1}<-1+\sqrt{2},-1+\sqrt{2} \leq a_{1}<1$ or $a_{1}=\frac{11}{5}$, then the real entries are two, that is: $\pm \sqrt{r_{21}}$, $\pm \sqrt{r_{23}}$ or $\pm \sqrt{\frac{3}{23}}$, respectively.

d) For $a_{1}=1$, there not exist real strange fixed points.

9. When $0<b_{2}<\frac{1}{90}(33-7 \sqrt{21})$, the number of strange fixed points depends on the relation among $a_{1}$ and the roots of $p_{1}(t)$ and $p_{2}(t)$, previously defined.

a) If $a_{1}<r_{11}$ or $a_{1}>\frac{-88}{-40+18 b_{2}+b_{2}^{2}}$, there are six different entries for the strange fixed points $\pm \sqrt{r_{21}}, \pm \sqrt{r_{22}}, \pm \sqrt{r_{23}}$.

b) For $a_{1}=r_{11}, a_{1}=\frac{-88}{-40+18 b_{2}+b_{2}^{2}}$ or $r_{14}<a_{1}<\frac{-88}{-40+18 b_{2}+b_{2}^{2}}$, there are four real entries: $\pm \sqrt{r_{21}}, \pm \sqrt{r_{22}}$ in the first two cases and $\pm \sqrt{r_{22}}, \pm \sqrt{r_{23}}$ in the last one.

c) If $r_{11}<a_{1} \leq 0$ or $a_{1}=r_{14}$, there only exist two real entries, $\pm \sqrt{r_{21}}$ and $\pm \sqrt{r_{22}}$ respectively.

d) When $0<a_{1}<r_{14}$, there are not strange fixed points.

10. If $\frac{1}{90}(33-7 \sqrt{21})<b_{2}<\frac{4}{63}(44 \sqrt{22}-205)$, the following cases appear:

a) For $a_{1}<r_{11}$ or $a_{1}>\frac{-88}{-40+18 b_{2}+b_{2}^{2}}$, there are six real entries of the strange fixed points: $\pm \sqrt{r_{21}}, \pm \sqrt{r_{22}}, \pm \sqrt{r_{23}}$.

b) If $a_{1}=r_{11}, a_{1}=\frac{-88}{-40+18 b_{2}+b_{2}^{2}}$ or $r_{12}<a_{1}<\frac{-88}{-40+18 b_{2}+b_{2}^{2}}$, four real entries exist: $\pm \sqrt{r_{21}}, \pm \sqrt{r_{22}}$ in the first two cases and $\pm \sqrt{r_{22}}, \pm \sqrt{r_{23}}$ in the last one.

c) When $r_{11}<a_{1} \leq 0$ or $a_{1}=r_{12}$, only two real entries appear: $\pm \sqrt{r_{21}}$ or $\pm \sqrt{r_{22}}$, respectively.

d) For $0<a_{1}<r_{12}$, there not exist strange fixed points.

11. If $b_{2}=\frac{4}{63}(44 \sqrt{22}-205)$, then some cases appear:

a) For $a_{1}<r_{11}$ or $a_{1}>\frac{-88}{-40+18 b_{2}+b_{2}^{2}}$, the number of real entries of the strange fixed points is six, being $\pm \sqrt{r_{21}}, \pm \sqrt{r_{22}}, \pm \sqrt{r_{23}}$.

b) If $a_{1}=r_{11}$, then four different real entries exist: $\pm \sqrt{r_{21}}, \pm \sqrt{r_{22}}$.

c) When $r_{11}<a_{1} \leq 0$ or $a_{1}=\frac{-88}{-40+18 b_{2}+b_{2}^{2}}$, there are only two real entries $\pm \sqrt{r_{21}}$.

d) Finally, if $0<a_{1}<\frac{-88}{-40+18 b_{2}+b_{2}^{2}}$ then there are not strange fixed points.

12. For values of $b_{2}$ in the interval $\frac{4}{63}(44 \sqrt{22}-205)<b_{2}<\frac{1}{4}$, different cases are analyzed:

a) If $a_{1}<r_{11}$ or $a_{1}>r_{12}$, there exist six real entries for the strange fixed points, $\pm \sqrt{r_{21}}, \pm \sqrt{r_{22}}, \pm \sqrt{r_{23}}$.

b) For $a_{1}=r_{11}$ or $a_{1}=r_{12}$, four real entries appear $\pm \sqrt{r_{21}}$ and $\pm \sqrt{r_{22}}$ or $\pm \sqrt{r_{23}}$, respectively. 
c) When $r_{11}<a_{1} \leq 0$ or $\frac{-88}{-40+18 b_{2}+b_{2}^{2}}<a_{1}<r_{12}$, there are only two real entries, $\pm \sqrt{r_{21}}$.

d) If $0<a_{1} \leq \frac{-88}{-40+18 b_{2}+b_{2}^{2}}$, there are not strange fixed points.

13. When $b_{2}=\frac{1}{4}$, the relation among parameter $a_{1}$ and the first root of $p_{5}(t)=686 t^{3}+245 t^{2}-1792 t+1024$, denoted by $r_{51}$ and the roots of $p 2(t)$, denoted by $r_{2 j} j=1,2,3$, is a key fact in the number of real entries of the strange fixed points.

a) If $a_{1}<r_{51}$ then there exist six real different entries, $\pm \sqrt{r_{21}}, \pm \sqrt{r_{22}}, \pm \sqrt{r_{23}}$.

b) For $a_{1}=r_{51}$ there are four real entries $\pm \sqrt{r_{21}}$ and $\pm \sqrt{r_{22}}$.

c) When $a_{1}<r_{51} \leq 0$ or $a_{1}>\frac{1408}{567}$, then the number of real entries reduces to two: $\pm \sqrt{r_{21}}$.

d) If $0<a_{1} \leq \frac{1408}{567}$, then there are not strange fixed points.

14. If $\frac{1}{4}<b_{2}<\frac{1}{90}(33+7 \sqrt{21})$, the following cases are analyzed to deduce the number of strange fixed points.

a) If $a_{1}<r_{11}, r_{12}<a_{1} \leq 0$ or $a_{1}>\frac{-88}{-40+18 b_{2}+b_{2}^{2}}$, then there are two real entries, $\pm \sqrt{r_{21}}$.

b) For $a_{1}=r_{11}$ or $a_{1}=r_{12}$, the number of real entries is four, $\pm \sqrt{r_{21}}$ and $\pm \sqrt{r_{23}}$ or $\pm \sqrt{r_{22}}$, respectively.

c) On the other hand, if $r_{11}<a_{1}<r_{12}$ then there are six real entries: $\pm \sqrt{r_{21}}, \pm \sqrt{r_{22}}, \pm \sqrt{r_{23}}$.

d) If $0<a_{1} \leq \frac{-88}{-40+18 b_{2}+b_{2}^{2}}$, then there are not strange fixed points.

15. When $\frac{1}{90}(33+7 \sqrt{21}) \leq b_{2} \leq 2$, the analysis is simpler:

a) For $b_{2}<2, a_{1} \leq 0$ or $a_{1}>\frac{-88}{-40+18 b_{2}+b_{2}^{2}}$, the only real entries are $\pm \sqrt{r_{21}}$.

b) If $b_{2}=2$ or if $b_{2}<2$ and $0<a_{1} \leq \frac{-88}{-40+18 b_{2}+b_{2}^{2}}$, there not exist strange fixed points.

16. When $b_{2}>2$, the number of cases is also reduced:
a) If $a_{1}<\frac{-88}{-40+18 b_{2}+b_{2}^{2}}$ or $a_{1} \geq 0$, the only real entries of the strange fixed points are $\pm \sqrt{r_{21}}$.
b) When $\frac{-88}{-40+18 b_{2}+b_{2}^{2}} \leq a_{1}<0$ there are not strange fixed points.

\subsection{Stability analysis of the fixed points}

To check the stability of the fixed points we need to calculate the Jacobian matrix and evaluate their eigenvalues in each fixed point,

$$
J\left(x_{1}, x_{2}\right)=\left(\begin{array}{cc}
J_{1} & 0 \\
0 & J_{2}
\end{array}\right)
$$

where

$$
J_{j}=-\frac{\left(x_{j}-1\right)^{3}\left(x_{j}+1\right)^{3} s_{j}}{32 x_{j}^{6}\left(a_{1} b_{2} x_{j}^{2}-a_{1} b_{2}-2 a_{1} x_{j}^{2}+2 a_{1}+4 x_{j}^{2}\right)^{2}}
$$

and

$$
\begin{aligned}
s_{j}= & -5 a_{1}^{2} b_{2}^{3}+20 a_{1}^{2} b_{2}^{2}-20 a_{1}^{2} b_{2}+x_{j}^{2}\left(4 a_{1}^{2} b_{2}^{3}-4 a_{1}^{2} b_{2}^{2}-32 a_{1}^{2} b_{2}+48 a_{1}^{2}+28 a_{1} b_{2}^{2}-32 a_{1} b_{2}-48 a_{1}\right) \\
& +x_{j}^{4}\left(a_{1}^{2} b_{2}^{3}-16 a_{1}^{2} b_{2}^{2}+52 a_{1}^{2} b_{2}-48 a_{1}^{2}+4 a_{1} b_{2}^{2}-96 a_{1} b_{2}+176 a_{1}-160\right)
\end{aligned}
$$

with eigenvalues $\alpha_{j}=J_{j}, j=1,2$. It is clear that by evaluating these eigenvalues in the roots of the polynomial we obtain $J_{j}( \pm 1)=0, j=1,2$ which means that all the roots are attractive.

The stability of the rest of fixed points is specially tedious for analyzing, as the intervals (the domain of the parameters $a_{1}$ and $b_{2}$ ) where the strange fixed points are real must be taken into account. For the sake of simplicity, we study the case $b_{2}=0$.

As it was stated in the previous section, there are at most six different components of the strange fixed points depending on $a_{1}$, so the different cases where the number of real strange fixed points change must be detailed. To check their stability we will draw $2 \mathrm{D}$ stability diagrams, that consist of plotting the curves described by $\left|J_{j}\left(r_{i}\right)\right| j=1,2, i \in\{1,2, \ldots, 6\}$ respect the values of parameter $a_{1}$. 
Theorem 2 The stability of the real strange fixed points of vectorial fixed point operator associated to OstrowskiChun family for the case $b_{2}=0$ is:

i) All the strange fixed points whose components belong to $\left\{\sqrt{r_{22}}, \pm 1\right\}$ are attractive if $-1.9809<a_{1}<-1.981$ or $0.91415<a_{1}<0.9196$.

ii) Strange fixed points of entries belonging to $\left\{\sqrt{r_{23}}, \pm 1\right\}$ are attractive for $\frac{11}{5}<a_{1}<2.4899$.

iii) The rest of strange fixed points are repulsive or saddle.

Summarizing, for values of $a_{1}$ established in cases $\left.i\right)$ and ii) there are 12 attractive strange fixed points, 24 repulsive and 24 saddle. For values of $a_{1}$ out of these regions there are 36 repulsive fixed points and 24 are saddle.

An sketch of the proof is presented in what follows. To prove the theorem we need to draw 2D stability diagrams for the components $r_{2 j}$ of the strange fixed points. Let us remark that $r_{21}=0$ and $J_{j}(0)$ is not defined for $j=1,2$. So, the stability of this point must be deduced from the bifurcation diagram.

Stability diagrams show the absolute value of $J_{j}, j=1,2$ on a component of an strange fixed point. As the operator and its eigenvalues have symmetry about $x$ and $y$ axis we will only use the positive square roots of the zeros of $p_{2}(t)$, that is $\sqrt{r_{21}}, \sqrt{r_{22}}$ and $\sqrt{r_{23}}$. That means if we have 3 attractive fixed points in the first quadrant we will have total 12 attractive strange fixed points.

The stability of the strange fixed points must be studied taking into account all the different subintervals where the number of real fixed points varies:

a) If $a_{1}<-1-\sqrt{2}$, both points satisfy $\left|J_{j}\left(\sqrt{r_{2 i}}\right)\right|>1$ for $j=1,2, i=2,3$ as can be observed in Figure 1 . The same behavior is observed for $\sqrt{r_{22}}$ and $\sqrt{r_{23}}$ in the interval $1<a_{1}<\frac{11}{5}$.

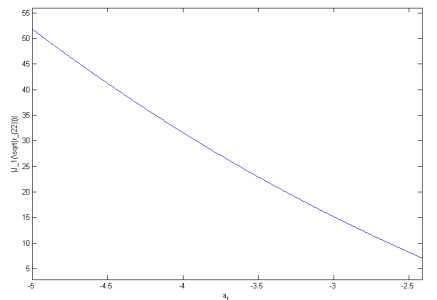

(a) $\left|J_{j}\left(\sqrt{r_{22}}\right)\right|$

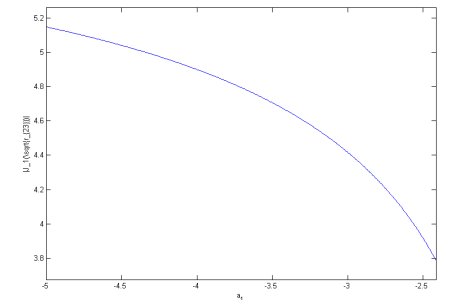

(b) $\left|J_{j}\left(\sqrt{r_{23}}\right)\right|$

Fig. 1: Stability diagrams for $\sqrt{r_{22}}$ and $\sqrt{r_{23}}$ with $b_{2}=0$ and $a_{1}<-1-\sqrt{2}$

b) For $a_{1}>\frac{11}{5}$, then $\left|J_{j}\left(\sqrt{r_{22}}\right)\right|>1 j=1,2$ meanwhile $\left|J_{j}\left(\sqrt{r_{23}}\right)\right|<1$ in the interval $\frac{11}{5}<a_{1}<2.4899$, as can be observed in Figure 2.

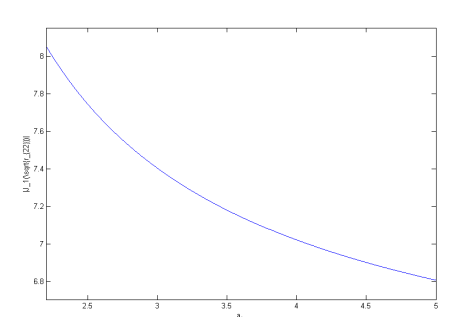

(a) $\left|J_{j}\left(\sqrt{r_{22}}\right)\right|$

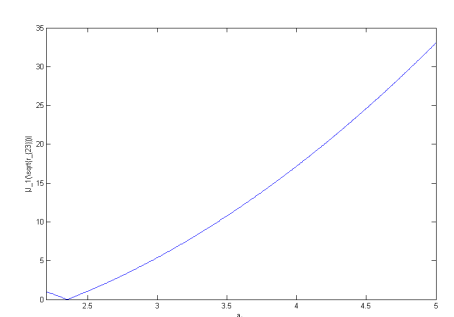

(b) $\left|J_{j}\left(\sqrt{r_{23}}\right)\right|$

Fig. 2: Stability diagrams for $\sqrt{r_{22}}$ and $\sqrt{r_{23}}$ with $b_{2}=0$ and $a_{1}>\frac{11}{5}$ 
c) If $-1-\sqrt{2}<a_{1}<-1+\sqrt{2},\left|J_{j}\left(\sqrt{r_{23}}\right)\right|>1$ and $\left|J_{j}\left(\sqrt{r_{22}}\right)\right|<1$ in the interval $-1.9809<a_{1}<-1.981$. This behavior can be observed in Figure 3 .

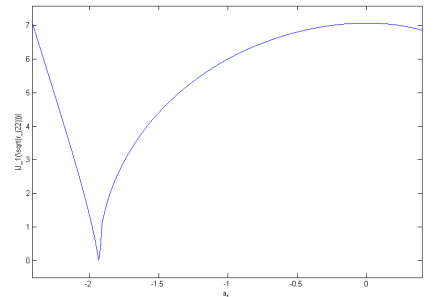

(a) $\left|J_{j}\left(\sqrt{r_{22}}\right)\right|$

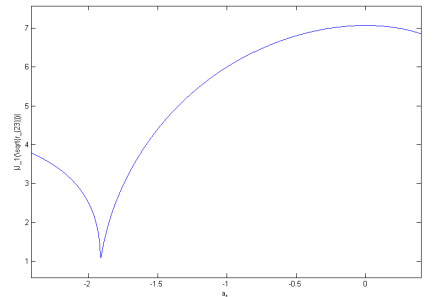

(b) $\left|J_{j}\left(\sqrt{r_{23}}\right)\right|$

Fig. 3: Stability diagrams for $\sqrt{r_{22}}$ and $\sqrt{r_{23}}$ with $b_{2}=0$ and $-1-\sqrt{2}<a_{1}<-1+\sqrt{2}$

d) When $-1+\sqrt{2} \leq a_{1}<1$ then $\mid J_{j}\left(\sqrt{r_{23}} \mid>1\right.$ and $\mid J_{j}\left(\sqrt{r_{22}} \mid<1\right.$ in the interval $0.91415<a_{1}<0.9196$ (see Figure 4).

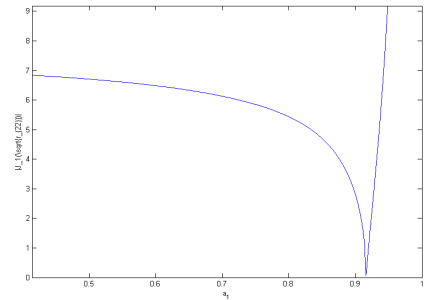

(a) $\left|J_{j}\left(\sqrt{r_{22}}\right)\right|$

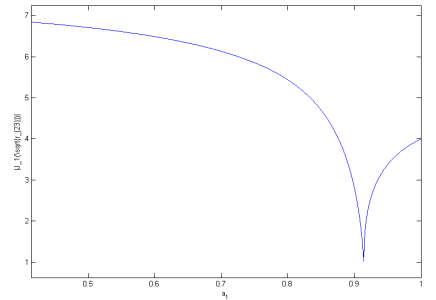

(b) $\left|J_{j}\left(\sqrt{r_{23}}\right)\right|$

Fig. 4: Stability diagrams for $r_{22}$ and $r_{23}$ with $b_{2}=0$ and $-1+\sqrt{2} \leq a_{1}<1$

\subsection{Bifurcation diagrams}

Bifurcation diagrams show the behavior of a method when the initial estimation is near a strange fixed point. To study the bifurcation phenomena, we use Feigenbaum diagrams of each coordinate function of fixed point function $\lambda_{j}^{p}(x), j=1,2$ by using as a starting point each one of the strange fixed points of the map and observing the ranges of the parameter $a_{1}$ where changes of stability or other behaviors happen. As both coordinate functions coincide, it is enough to represent the "scalar" bifurcation diagram of one of the coordinate function on each possible component of the strange fixed point.

To draw these Feigenbaum diagrams, 500 elements of the orbit of each strange fixed point are calculated, plotting the last 200 , for each value of parameter $a_{1}$ (after a partition of the analyzed interval in 5000 subintervals).

In the previous section it was analyzed the stability of these points and in Figure 5 the diagrams corresponding to $r_{3}=\sqrt{r_{22}}, r_{4}=-\sqrt{r_{22}}, r_{5}=\sqrt{r_{23}}$ and $r_{6}=\sqrt{r_{23}} i=3,4,5,6$ are presented. The reason why the diagrams of $r_{1}$ and $r_{2}$ do not appear is that the coordinate functions of the fixed point operator are not defined on $\pm \sqrt{r_{21}}=0$. However, its stability can be deduced from the rest of bifurcation diagrams.

As the abscissas axis correspond to the value of the parameter $a_{1}$ and the ordinate axis show the value of the last hundred iterations (of a total of 500), the first observed aspect is the symmetry respect $a_{1}$ axis. Iterates can converge to 1 and -1 , components of the roots of $p(x)$, or to attracting strange fixed points (including the zero, that is attracting for $\left.a_{1} \in(1,2)\right)$, for the parameter defined in the intervals obtained in Theorem 2 , or to other attracting structures. 


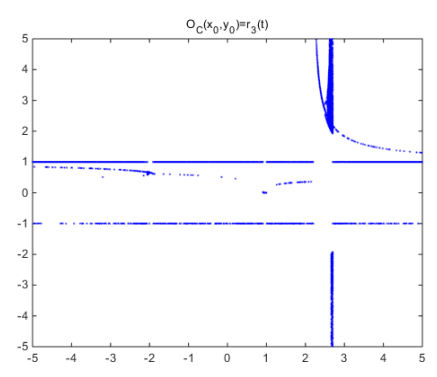

(a) $r_{3}$

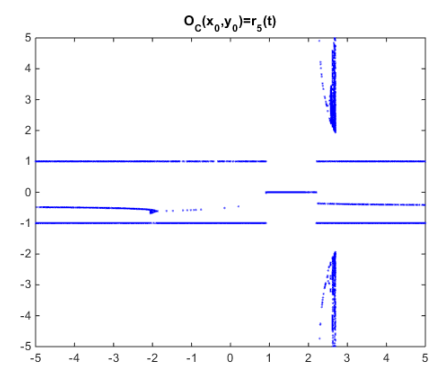

(c) $r_{5}$

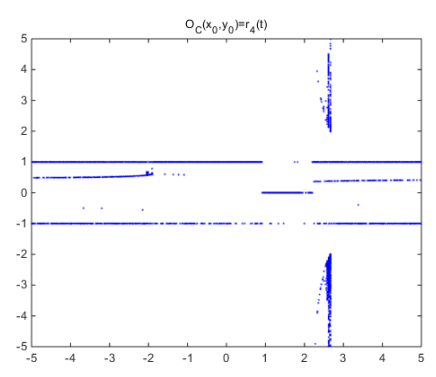

(b) $r_{4}$

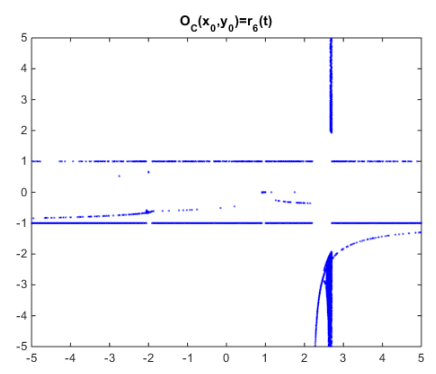

(d) $r_{6}$

Fig. 5: Bifurcation diagrams for $b_{2}=0$

In Figure 6, some details of diagrams of $r_{4}$ and $r_{5}$ (appearing in Figures $5 \mathrm{~b}$ and $5 \mathrm{c}$ ) are showed. In them, it is observed that, when an attracting fixed point changes its stability, period-doubling bifurcation appears yielding cascades of attracting periodic orbits of period $2,4,8, \ldots$ Then, in blue regions, chaos appears. In the following section some of these periodic cases appear in the dynamical planes; also strange attractors appear in some of these blue regions and they also will be found.

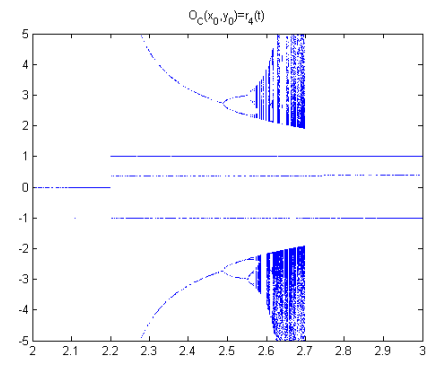

(a) $r_{4}$

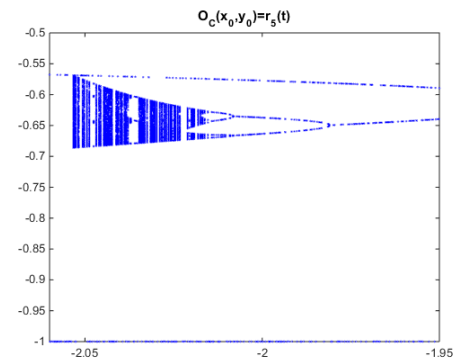

(b) $r_{5}$

Fig. 6: Some details of bifurcation diagrams corresponding to $r_{4}$ and $r_{5}$

\section{Convergence and Dynamical planes of the family}

Convergence planes (see [11]) are a useful tool to check the behavior of a family of iterative methods near strange fixed points. The convergence space is obtained by associating each point of the plane with real values of $a_{1}$ (axis OX) and $b_{2}$ (axis OY). We have used $800 \times 800$ different combinations of $a_{1}$ and $b_{2}$. The points of the plane shown in black correspond to the parameter values for which the associated iterative method does not converge to a root with a tolerance of $10^{-3}$ after 500 iterations, taking as the initial estimate every strange fixed point. Points shown in red converge to a root of the polynomial. 


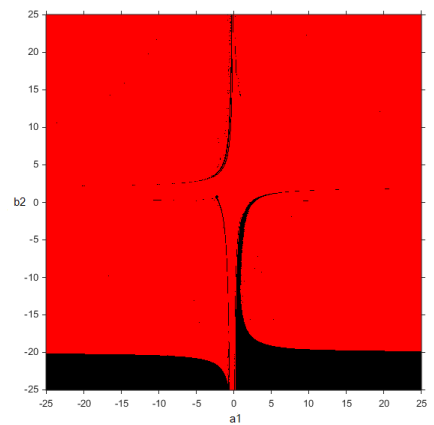

(a)

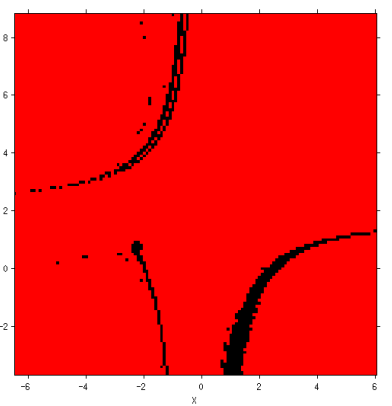

(b)

Fig. 7: Convergence plane and a detail of its central region

A dynamical plane is a visual representation of a method that gives qualitative information about its behavior. Dynamical planes are built by applying the iterative method with different initial estimations distributed in a mesh. If the numerical method converges to a root of the system the point of the plane is painted in different colors depending on the root they converge to, while the points painted in black mean no convergence to any root after 40 iterations.

The dynamical planes of this manuscript have been obtained by using $400 \times 400$ subintervals, a maximum of 40 iterations and an error estimation of $10^{-3}$, when the iterates tend to a fixed point.

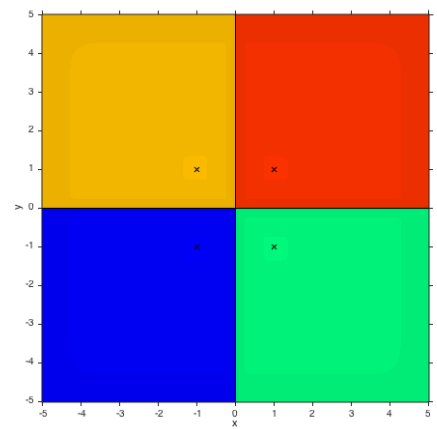

(a) Ostrowski

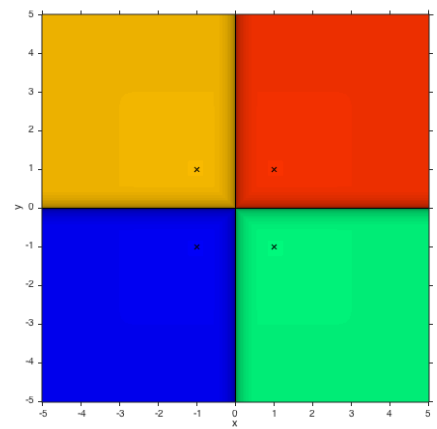

(b) Chun

Fig. 8: Dynamical planes for Ostroswki' and Chun's methods on $p(x)$

Figure 8 shows the dynamical plane for Ostrowski' and Chun's schemes on $p(x)$. We can see four basins of attraction, one for each root of the polynomial. Both methods show a stable behavior, as the only fixed points with a basin of attraction are the roots of the polynomial.

Other methods don't show a stable behavior if any strange fixed point is attractive, as we can see in Figure 9 , that shows two combination of parameters that have 12 attractive fixed points.

The stability of a method depends not only on the existence of attracting strange fixed points but also on possible attracting periodic orbits. Figure 10 shows a combination of parameters that lead to six periodic orbits. In Figure 10 we have modified the dynamical plane program to paint in different colors the points that tend to every periodic orbit.

For $b_{2}=0$ the bifurcation diagram shows blue chaotic regions where strange attractors can appear, for some values of $a_{1}$. To visualize strange attractors we use a 2-D plot, in which parameter $a_{1}$ is fixed in a blue region value and the asymptotic behavior of the iterative method with many initial estimatations are shown. In Figure 11a, a dynamical plane showing chaotic behavior is shown, for the method $a_{1}=2.59$.

In order to get more details about this chaotic element, we plot in Figure 11b the iteration of the multidimensional fixed point operator of the family, for a value of parameter $a_{1}$ in one of the blue chaotic regions of Figure 5 close to $a_{1}=2.5$. So, symmetric strange attractors have been found, (see Figure 11b). The way these 


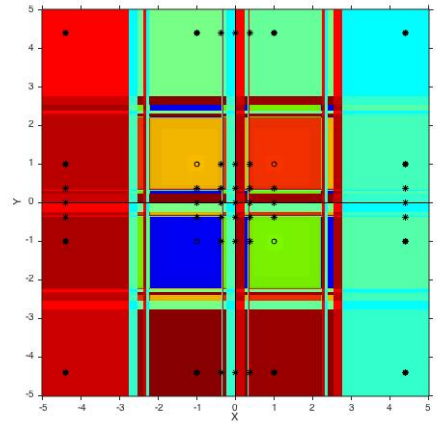

(a) $a_{1}=2.3, b_{2}=0$

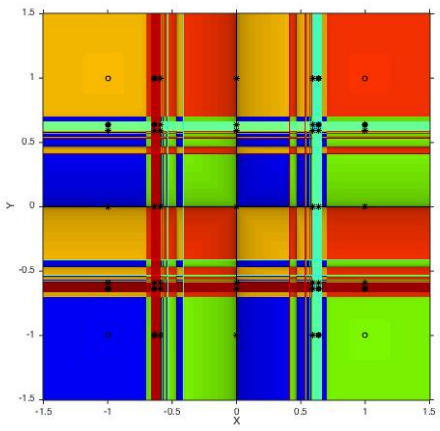

(b) $a_{1}=-1.95, b_{2}=0$

Fig. 9: Dynamical planes with attracting fixed points

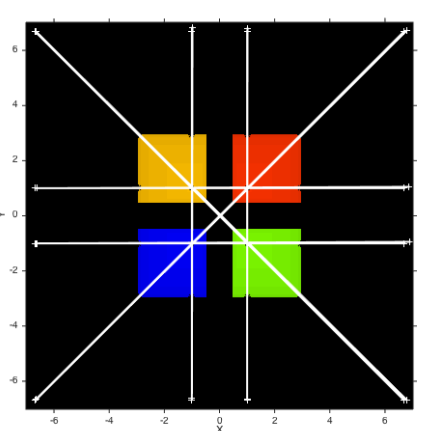

(a)

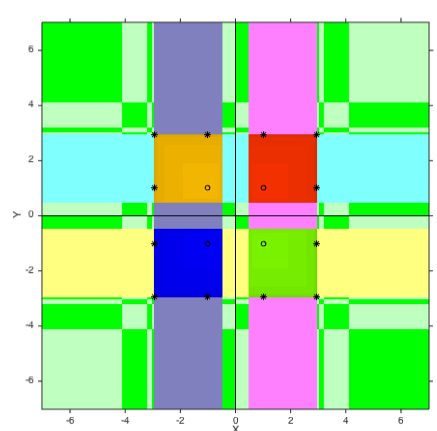

(b)

Fig. 10: Dynamical planes for the method $a_{1}=-2.5, b_{2}=3.7$

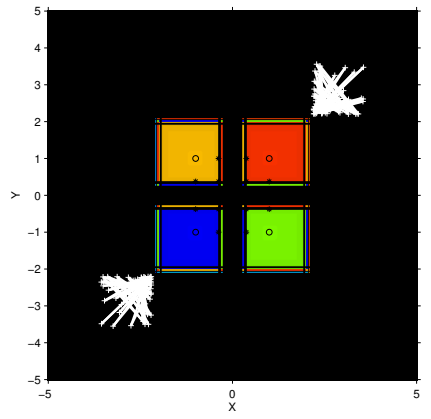

(a) Dynamical plane

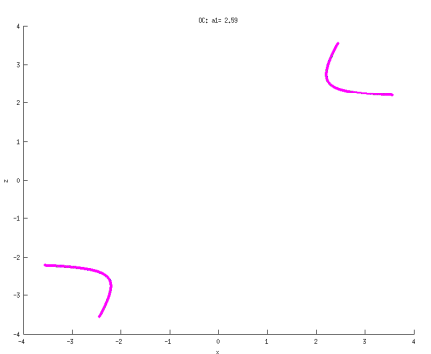

(b) Strange attractors

Fig. 11: Dynamical plane and strange attractors for the method $a_{1}=2.59, b_{2}=0$

pictures have been obtained is the following: fixing the value of parameter $a_{1}, 10000$ different initial estimations have been taken in a small rectangle close to the origin. The method has been used on each of them, plotting one point per iteration. The resulting image show how the some attracting strange fixed points appearing in the bifurcation diagrams change into attracting regions. However, the set of initial estimations that belong to their respective basins of attraction is very reduced, as well as the interval of real values of $a_{1}$ that induces this behavior. 


\section{Numerical tests}

In this section, we are going to apply different elements of the proposed family for solving the nonlinear system obtained by using the divided differences technique for approximating the solution of a diffusion problem with may applications in several fields. We use members of (2) with stable properties and other ones with bad dynamical behavior, following the results obtained in the previous section.

Fisher's equation

$$
u_{t}=D u_{x x}+r u\left(1-\frac{u}{k}\right)
$$

was proposed by Fisher [8] as a model of diffusion in population dynamics, where $D>0$ is the diffusion constant, $r$ is the growth rate of the species and $k$ is the carrying capacity. In recent years, the equation has been used as a basis for a wide variety of models for the spatial spread of genes in a population. This equation has also interesting applications in chemical waves propagation, financial mathematics and economics (it estimates the relationship between nominal and real interest rates inflation).

We analyze a particular case of Fisher's equation, corresponding to $D=k=r=1, x \in[-25,50]$, the boundary conditions $u(-25, t)=1, u(50, t)=0, t>0$, and the initial condition

$$
u(x, 0)= \begin{cases}1, & x<-10 \\ 0, & -10 \leq x \leq 10 \\ 1 / 4, & 10<x<20 \\ 0, & x \geq 20\end{cases}
$$

By applying an implicit method of finite differences we transform problem (5) in a family of nonlinear systems, which provides the approximated solution in a time $t_{k}$ from the approximated solution in $t_{k-1}$. We choose the spacial step $h=75 / n x$ and the temporal step $k=T_{\max } / n t$, where $n x$ and $n t$ are the number of $x$-subintervals and $t$-subintervals, respectively, and $T_{\max }$ is the final time of our study. So, we have selected a grid of domain $[-25,50] \times\left[0, T_{\max }\right]$ with points $\left(x_{i}, t_{j}\right)$,

$$
x_{i}=0+i h, \quad i=0,1, \ldots, n x, \quad t_{j}=0+j k, \quad j=0,1, \ldots, n t .
$$

We want to estimate the solution of (5) at these points, by transforming it in many nonlinear systems, as much as the number of $t_{j}$. To do that, we use the following approximations:

$$
\begin{aligned}
u_{t}(x, t) & \approx \frac{u(x, t)-u(x, t-k)}{k}, \\
u_{x x}(x, t) & \approx \frac{u(x+h, t)-2 u(x, t)+u(x-h, t)}{h^{2}},
\end{aligned}
$$

Denoting by $u_{i, j}$ the estimation of the solution at $\left(x_{i}, t_{j}\right)$ and by replacing them in $(5)$, we construct the following nonlinear system

$$
\frac{u_{i, j}-u_{i, j-1}}{k}=\frac{u_{i+1, j}-2 u_{i, j}+u_{i-1, j}}{h^{2}}+u_{i, j}-u_{i, j}^{2},
$$

for $i=1,2, \ldots, n x-1$ and $t=1,2, \ldots, n t$. After some algebraic manipulations we transform this system in:

$$
k u_{i+1, j}+\left(k h^{2}-2 k-h^{2}\right) u_{i, j}-\left(k h^{2}\right) u_{i, j}^{2}+k u_{i-1, j}=-h^{2} u_{i, j-1}
$$

for $i=1,2, \ldots, n x-1$ and $j=1,2, \ldots, n t$. For a fixed $j$, we have the following nonlinear system of size $(n x-1) \times(n x-1):$

$$
\begin{aligned}
& k u_{2, j}+\left(k h^{2}-2 k-h^{2}\right) u_{1, j}-k h^{2} u_{1, j}^{2}=-h^{2} u_{1, j-1}-k, \\
& k u_{i+1, j}+\left(k h^{2}-2 k-h^{2}\right) u_{i, j}-k h^{2} u_{i, j}^{2}+k u_{i-1, j}=-h^{2} u_{i, j-1}, i=2,3, \ldots, n x-2, \\
& \left(k h^{2}-2 k-h^{2}\right) u_{n x-1, j}-k h^{2} u_{n x-1, j}^{2}+k u_{n x-2, j}=-h^{2} u_{1, j-1}-k .
\end{aligned}
$$

The unknowns of this system are $u_{1, j}, u_{2, j}, \ldots, u_{n x-1, j}$, that is, the approximations of the solution in $t_{j}$. We observe that for solving this system we need the solution in $t_{j-1}$.

We are going to solve this system for different values of $T_{\max }$, using in each case $n x=20$ and methods M1 $(a=b=1)$, M2 (Chun's method), M3 $(a=2.3, b=0)$ and M4 that corresponds to $a=-2.5 b=3.7$. The last two schemes have been selected in the black unstable region of the convergence plane showed in the previous section, meanwhile M1 and M2 correspond to the red stable region. As initial guess we use the approximated 
solution of the previous $t_{j}$. In Tables 1 and 2 , we calculate the approximated solution at different $T_{\max }$ showing $\left\|F\left(x^{(k+1)}\right)\right\|$, the mean number of iterations needed for solving the nonlinear systems appearing for each instant $t_{j}$ in the whole process and the elapsed time, in order to analyze the stability and consistence of the new method.

All computations are performed using variable precision arithmetics with 500 digits of mantissa. For every value of $T_{\max }$, we analyze the number of iterations (iter) needed to converge to the solution such that $\left\|F\left(x^{(k+1)}\right)\right\|<10^{-12}$ is satisfied, where $\|\cdot\|$ denotes the Euclidean norm.

\begin{tabular}{|c|c|c|c|c|c|}
\hline \multicolumn{2}{|c|}{$T_{\max }=0.1$} & M1 & M2 & M3 & M4 \\
\hline \multirow[t]{4}{*}{$n t=100$} & iter & 1 & 2 & 2 & 1 \\
\hline & $\left\|F\left(x^{(k+1)}\right)\right\|$ & $1.61 \mathrm{e}-13$ & $3.39 \mathrm{e}-24$ & $3.68 \mathrm{e}-22$ & $1.52 \mathrm{e}-13$ \\
\hline & e-time & 94.65 & 171.98 & 509.57 & 283.30 \\
\hline & iter & 1 & 2 & $\mathrm{nc}$ & 1 \\
\hline \multirow{2}{*}{$n t=500$} & $\left\|F\left(x^{(k+1)}\right)\right\|$ & $2.57 \mathrm{e}-16$ & $3.64 \mathrm{e}-31$ & & $2.54 \mathrm{e}-16$ \\
\hline & e-time & 464.85 & 856.44 & & 1405.43 \\
\hline \multicolumn{2}{|c|}{$T_{\max }=0.3$} & M1 & M2 & M3 & M4 \\
\hline \multirow[t]{3}{*}{$n t=100$} & iter & 2.01 & 2 & 2 & 2 \\
\hline & $\left\|F\left(x^{(k+1)}\right)\right\|$ & $2.21 \mathrm{e}-29$ & $2.21 \mathrm{e}-29$ & $1.03 \mathrm{e}-18$ & $1.88 \mathrm{e}-29$ \\
\hline & e-time & 492.92 & 175.31 & 513.66 & 547.91 \\
\hline \multirow[t]{3}{*}{$n t=500$} & iter & 1 & 1 & 2 & 1.002 \\
\hline & $\left\|F\left(x^{(k+1)}\right)\right\|$ & $2.23 \mathrm{e}-18$ & $2.25 \mathrm{e}-14$ & $1.31 \mathrm{e}-23$ & $2.12 \mathrm{e}-14$ \\
\hline & e-time & 437.80 & 1221.66 & 2384.80 & 1375.31 \\
\hline \multicolumn{2}{|c|}{$T_{\max }=0.5$} & M1 & M2 & M3 & M4 \\
\hline \multirow[t]{3}{*}{$n t=100$} & iter & 2.01 & 2 & 2 & 2 \\
\hline & $\left\|F\left(x^{(k+1)}\right)\right\|$ & $4.02 \mathrm{e}-27$ & $3.99 \mathrm{e}-27$ & $4.32 \mathrm{e}-17$ & $5.06 \mathrm{e}-27$ \\
\hline & e-time & 496.80 & 554.01 & 554.01 & 581.26 \\
\hline \multirow[t]{3}{*}{$n t=500$} & iter & 1.002 & 2 & 2 & 1.0060 \\
\hline & $\left\|F\left(x^{(k+1)}\right)\right\|$ & $1.82 \mathrm{e}-13$ & $5.52 \mathrm{e}-22$ & $5.52 \mathrm{e}-22$ & $1.56 \mathrm{e}-13$ \\
\hline & e-time & 1322.68 & 2587.08 & 2587.08 & 1502.13 \\
\hline
\end{tabular}

Table 1: Numerical results for different values of $T_{\max }$

\begin{tabular}{|c|c|c|c|c|c|}
\hline \multicolumn{2}{|c|}{$T_{\max }=0.7$} & M1 & M2 & M3 & M4 \\
\hline \multirow[t]{3}{*}{$n t=100$} & iter & 2.0100 & 2 & 2 & 2 \\
\hline & $\left\|F\left(x^{(k+1)}\right)\right\|$ & $1.17 \mathrm{e}-25$ & $1.15 \mathrm{e}-25$ & $4.95 \mathrm{e}-16$ & $7.06 \mathrm{e}-16$ \\
\hline & e-time & 540.12 & 527.59 & 543.90 & 495.37 \\
\hline \multirow[t]{3}{*}{$n t=500$} & iter & $\mathrm{nc}$ & $\mathrm{nc}$ & $\mathrm{nc}$ & $\mathrm{nc}$ \\
\hline & $\left\|F\left(x^{(k+1)}\right)\right\|$ & - & - & - & - \\
\hline & e-time & - & - & - & - \\
\hline \multicolumn{2}{|c|}{$T_{\max }=1$} & M1 & M2 & M3 & M4 \\
\hline \multirow[t]{3}{*}{$n t=100$} & iter & 2.01 & 2 & 2 & 2 \\
\hline & $\left\|F\left(x^{(k+1)}\right)\right\|$ & $3.48 \mathrm{e}-24$ & $3.39 \mathrm{e}-24$ & $5.83 \mathrm{e}-15$ & $8.31 \mathrm{e}-15$ \\
\hline & e-time & 519.36 & 521.74 & 536.57 & 509.08 \\
\hline \multirow[t]{3}{*}{$n t=500$} & iter & 2.002 & 2 & 2 & 2 \\
\hline & $\left\|F\left(x^{(k+1)}\right)\right\|$ & $3.75 \mathrm{e}-31$ & $3.64 \mathrm{e}-31$ & $7.46 \mathrm{e}-20$ & $1.07 \mathrm{e}-19$ \\
\hline & e-time & 2489.67 & 2464.47 & 2631.47 & 2424.11 \\
\hline
\end{tabular}

Table 2: Numerical results for different values of $T_{\max }$

Let us remark that, as can be seen at Tables 1 and 2, the number of temporal subintervals do not need to be increased when $T_{\max }$ is higher. Moreover, due to the high order of convergence of the methods, the mean number of required iterations is very small, as well as the value of $\left\|F\left(x^{(k+1)}\right)\right\|$. Respect to the stability of the methods, it is noticed that methods M1 and M2 have similar behavior, meanwhile M3 and M4 take much more elapse time in converging, if they do it.

In Figure 12 we show the approximated solution of the problem when $T_{\max }=5$, by taking $n t=100$ and $n x=20$. It is observed that there not exist attenuation after this time, showing the expected behavior of a traveling wave. 


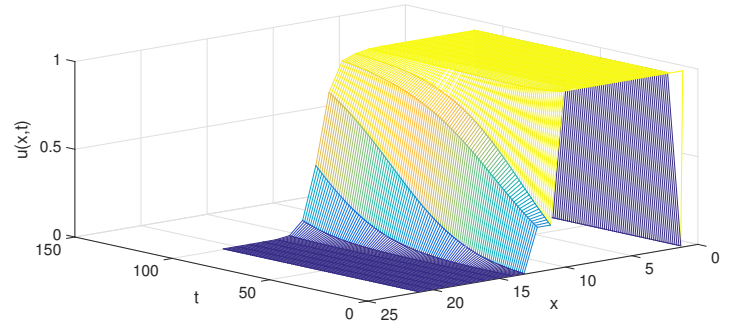

Fig. 12: Graphics of the approximated and exact solutions for $t \in[0,5]$ with $n t=100$

\section{Conclusions}

The dynamical analysis of the Ostrowski-Chun family gives us qualitative information about the stability and reliability of its elements. From this study, we can select the members of the class with good stability properties and avoid those with chaotic or unstable behavior. Both type of examples have been selected for solving the problem of traveling waves, confirming the information given by the dynamical study.

\section{References}

1. A. Cordero, J. García-Maimó, J. R. Torregrosa, M. P. Vassileva, Solving nonlinear problems by Ostrowski-Chun type parametric families, Journal of Mathematical Chemistry, 53, 430-449 (2015)

2. Á. A. Magreñán, Different anomalies in a Jarratt family of iterative root-finding methods, Applied Mathematics and Computation, 233, 29-38 (2014)

3. B. Neta, C. Chun, M. Scott, Basins of attraction for optimal eighth order methods to find simple roots of nonlinear equations, Applied Mathematics and Computation, 227, 567-592 (2014)

4. A. Cordero, J. García-Maimó, J. R. Torregrosa, M. P. Vassileva and P. Vindel, Chaos in King's iterative family, Applied Mathematics Letters, 26(8), 842-848 (2013)

5. A. Cordero, J.R. Torregrosa, F. Soleymani, Dynamical analysis of iterative methods for nonlinear systems or how to deal with the dimension?, Applied Mathematics and Computation, 244 398-412 (2014)

6. R.C. Robinson, An Introduction to Dynamical Systems, Continous and Discrete. Americal Mathematical Society, Providence (2012)

7. A. Cordero, J. García-Maimó, J. R. Torregrosa, M. P. Vassileva, Stability of a fourth order bi-parametric family of iterative methods, Journal of Computational and Applied Mathematics, doi: 10.1016/j.cam.2016.01.013 (2016)

8. R.A. Fisher, The wave of advance of advantageous genes, Ann. Eugenics, 7, 353-369 (1937)

9. M. Abad, A. Cordero, J.R. Torregrosa, A family of seventh-order schemes for solving nonlinear systems, Bull. Math. Soc. Sci. Math. Roumanie, 57(105), 133-145 (2014)

10. D. Budzko, A. Cordero, J.R. Torregrosa, A new family of iterative methods widening areas of convergence, Appl. Math. Comput., 252, 405-417 (2015)

11. A. Magreñan, A new tool to study real dynamics: The convergence plane, Applied Mathematics and Computation, 248, 215-224 (2014) 